\title{
A New Algorithm to Reduce the Mal-Operation of DOCR in Bi-directional Power Distribution Systems
}

\author{
Su-Hyeong Jang*, Joon-Seok Oh**, Ui-Yong Jeong** and Jae-Eon Kim ${ }^{\dagger}$
}

\begin{abstract}
DOCR can be used to efficiently increase the reliability and to protect the bi-directional D/L(Distribution Lines). As more DG(Distributed Generation)s attempt to connect the bi-directional $\mathrm{D} / \mathrm{L}$, there is an increasing need for studies of how to use DOCR installed in the $\mathrm{D} / \mathrm{L}$. This paper investigates the operating principles of DOCR and presents the results an effect of sequence and fault impedance in L-L(Line to Line) fault. An advanced DOCR operating algorithm is proposed to reduce the mal-operation of conventional DOCR. The proposed algorithm is applied to the bi-directional power flow system and shows that it decreases the mal-operation of DOCR through the computer simulation.
\end{abstract}

Keywords: Bi-directional power flow, Distributed generation, DOCR, Fault impedance, MalOperation, Sequence impedance

\section{Introduction}

The protective relay receives various signals like voltage and current as input data from instrument transformer(PT, $\mathrm{CT}$ ), numerically converts them, and is operated by comparing calculated values with specified threshold. It is essential for the power system to be stably operated, and required to have speed, selectivity, sensitivity, reliability, and economic feasibility as basic characteristics [1]. Also, countermeasures for networking, automation for the economic improvement of the system operation, and occurrence of the bi-directional power flow caused by the adoption of the DG(Distributed Generation) are increasingly required. Especially, introduction of DG causes protection coordination problems which were not shown in conventional radial distribution systems, and several researches for this are currently in progress.

Differential protective relaying, analysis of differences between d-q transformation calculated values before and after fault, distance protective relaying, DOCR(Directional Overcurrent Relaying), and others are being considered as technologies to counteract changes about protection caused by the adoption of DG. Particularly the DOCR is considered and applied as an effective method for the protection of the DG $[3,6,7]$. Generally two DOCR relays almost installed systems with DG. One is on the utility source side, the other is DG side. The setting of the relay needs very careful attention, because DOCR simultaneously identifies the magnitude and direction of fault current based by reference angle. Generally, accidents

\footnotetext{
$\dagger \quad$ Corresponding Author: School of Electric Engineering, Chungbuk National University, Korea. (pqdgs717@naver.com)

* R\&D Lab., LS Industrial Systems, Korea. (keumpyeon@naver.com)

** School of Electric Engineering, Chungbuk National Univ., Korea. ( \{jsoh, uyjeong\}@chungbuk.ac.kr)

Received: August 16, 2015; Accepted: December 8, 2015
}

related to the setting of DOCR relay account for $19 \%$ of the entire relay accident [8].

The operation principle of DOCR is systematically classified by W. K. Sonnemann in 1950, but contents of the related field mainly show only basic operation principle [1], [9]. Recent researches for the DOCR mainly focus studies about optimal DOCR coordination intending to minimize operation time when several DOCRs are operated at the same time, and complementation of DOCR operation in specific conditions.

Analysis methods like Trial and Error Approach, GA (Genetic Algorithm), PSO(Particle Swarm Optimizer), and others are considered [10] as an optimal DOCR coordination. But the solution of optimal DOCR coordination problems is based on mathematical algorithm, so application in actual power system field requires additional researches and long-term tests.

Methods that are proposed to complement problems regarding relative shortcoming of DOCR like SLF(Short Line Fault), HIF(High Impedance Fault), and others are mainly proposed by the manufacturer of the relay, but the awareness in the actual field is low [11-14].

A. Ukill provides simply structured DOCR only using current value [11]. However, there is a disadvantage that accurate protection is unable if faults occur in no-load status or extremely high current is loaded in a moment of fault. Method suggested by L. Susilo is to improve operation accuracy of DOCR by applying fuzzy algorithm, but generation of fuzzy rule reflecting various layer condition is not easy [12]. A. Wixon tries to enhance operation accuracy of DOCR with configuration of complex protection zone, but it is very narrowly applied because the amount of computing load of digital-relay is too high [13]. D. Yuan suggests method to combine DOCR with communication [14]. This method has high operation 
reliability but has disadvantage in terms of economic feasibility and maintenance of communication facility.

Thus, in this paper, the DOCR operation algorithm considering negative-sequence value occurring in fault is suggested in order to complement DOCR's problem (especially, in HIF condition). First, basic operation principle of DOCR is explained by using related calculus and DOCR relay model, and suggested new DOCR operation algorithm is detailedly described. $22.9[\mathrm{kV}]$ bidirectional distribution system is modeled with PSCAD/ EMTDC(Power System Computer Aided Design/ElectroMagnetic Transient Design and Control), and operation characteristics applying suggested DOCR algorithm are simulated.

\section{Basic Operation Principle of DOCR}

\subsection{Basic principle of DOCR}

The DOCR is operated by magnitude and phase of fault current. The DOCR detects line-to-line short fault(L-L fault), and DOCGR(Directional Overcurrent Ground Relaying) detects single line-to-ground fault(SLG fault). L-L fault current between phase $a \cdot b$ is able to be calculated as Eq. (1), (2) based on the generator model of Fig. 1 (Appendix 1)[15]. As it is L-L fault, zero-sequence component does not exist as shown in the Fig. 2. When fault is occurred, the system is balanced system.

$$
\begin{aligned}
& I_{a b}=\frac{\left(1-a^{2}\right) V_{a}}{Z_{1}+Z_{2}+Z_{f}}=\frac{\left(1-a^{2}\right) V_{a}}{\left(R_{1}+R_{2}+R_{f}\right)+j\left(X_{1}+X_{2}+X_{f}\right)} \\
& =A \angle \theta \\
& A=\frac{V_{a} \sqrt{G^{2}+H^{2}}}{2\left(M^{2}+N^{2}\right)} \\
& \theta=\tan ^{-1}\left(\frac{-(N+\sqrt{3} M)}{M-\sqrt{3} N}\right)
\end{aligned}
$$

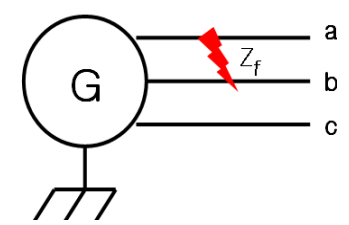

Fig. 1 Diagram of L-L fault

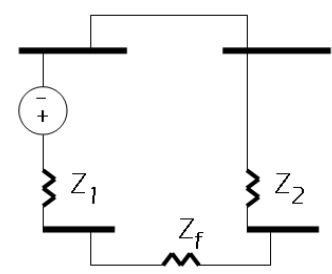

Fig. 2 Sequence diagram
$A, \theta$ : magnitude and angle of fault current

$$
\begin{aligned}
& M=R_{1}+R_{2}+R_{f}, \quad N=X_{1}+X_{2}+X_{f} \\
& G=(M-\sqrt{3} N) \quad H=(N+\sqrt{3} M)
\end{aligned}
$$

$Z_{1}$ : Positive Sequence Impedance

$Z_{2}:$ Negative Sequence Impedance

$Z_{f}:$ Fault Impedance

The phase angle $(\theta)$, main identification factor of DOCR, is determined by positive-sequence, negative-sequence and $\mathrm{X} / \mathrm{R}$ ratio of fault impedance through Eq. (1) and (2).

\subsection{Fault decision}

Difference between calculated phase angle of fault current and reference phase angle(polarization quantity) is used to decide phase angle $(\theta)$ component of DOCR. In this case, current.voltage on normal phase or positivesequence-zero-sequence angle are able to be used as reference phase angle, but line-to-line voltage on normal phase is mostly used on actual system. Table 1 shows reference phase angle of each fault type. For examples, phase angle of line voltage $\mathrm{V}_{\mathrm{bc}}$ is applied to detect L-L fault between phase $a \cdot b$ or phase $a \cdot c$.

It can be checked via Fig. 3 that $\mathrm{I}_{\mathrm{ab}}, \mathrm{I}_{\mathrm{ac}}$ which are case 1 are in trip region, but $I_{b c}$ is in non-trip region in condition that all impedances are inductive. For example, the $I_{a b}$ of Fig. 3 can be obtained by $R\left(=R_{1}+R_{2}+R_{f}\right)=0$, $\mathrm{X}\left(=\mathrm{X}_{1}+\mathrm{X}_{2}+\mathrm{X}_{\mathrm{f}}\right)=1$ and $\mathrm{V}_{\mathrm{a}}=1$ as p.u base in Eq. (1).

\subsection{Description of operation in bi-directional power flow condition}

In case that DOCR RY1 6 have been installed in Bidirectional power flow system like Fig. 4, when fault is occurred, the flow of protective operation is as follows. Time-delay setting of each relay is "RY1 $>$ RY $3>$ RY 5" and "RY6 > RY4 > RY2", and arrow-mark means protective direction.

Table 1. L-L fault type and polarization quantity

\begin{tabular}{c|c|c}
\hline Case & L-L fault type & Ref. angle (polarization quantity) \\
\hline 1 & $\mathrm{I}_{\mathrm{ab}}$ or $\mathrm{I}_{\mathrm{ac}}$ & $\angle\left(\mathrm{V}_{\mathrm{bc}}\right)-90 \sim \angle\left(\mathrm{V}_{\mathrm{bc}}\right)+90\left[^{\circ}\right]$ \\
\hline 2 & $\mathrm{I}_{\mathrm{ba}}$ or $\mathrm{I}_{\mathrm{bc}}$ & $\angle\left(\mathrm{V}_{\mathrm{ca}}\right)-90 \sim \angle\left(\mathrm{V}_{\mathrm{ca}}\right)+90\left[^{\circ}\right]$ \\
\hline 3 & $\mathrm{I}_{\mathrm{ca}}$ or $\mathrm{I}_{\mathrm{cb}}$ & $\angle\left(\mathrm{V}_{\mathrm{ab}}\right)-90 \sim \angle\left(\mathrm{V}_{\mathrm{ab}}\right)+90\left[^{\circ}\right]$ \\
\hline
\end{tabular}

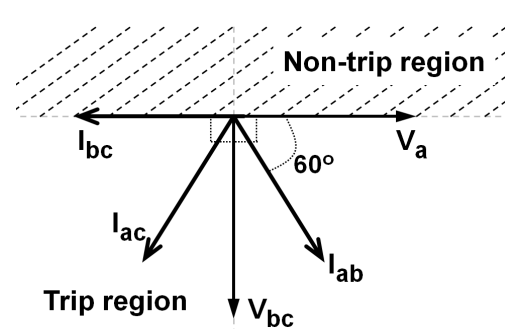

Fig. 3 'a'- phase directional unit response 


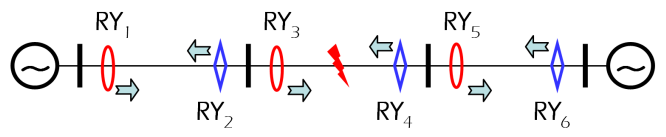

Fig. 4 Example of Bi-directional power flow system

(1) L-L fault is occurred,

(2) RY1·RY3 and RY4-RY6 simultaneously detects fault. (RY5 and RY2 are not operated by directional element)

(3) RY3 and RY4 are tripped in advance by Time-delay setting.

(4) Fault zone is decoupled, and power supply in normal sector is possible.

Thus, it is shown that the DOCR is confirmed to be appropriately operated in bi-directional D/L.

\section{Operation Characteristics of DOCR in Bi-directional Power System}

\subsection{Organization of the system}

As shown in the Fig. 5, 22.9[kV] bi-directional distribution system is composed of PSCAD(Appendix 2, 3) [3]. PSCAD does not support the DOCR relay model, so two types of model(v1, v2) are directly designed and implemented(Fig. 6, Table 2) using the programming language, and conditions of simulation are as follows.

○ v1 : Conventional DOCR model

o v2 : Advanced DOCR model proposed by this paper

$\circ$ RY1 $3 \cdot 5:$ Applied to the power A

○ RY2 $4 \cdot 6$ : Applied to the weak power B

$\circ$ All calculated phase angle is based on phase of $V_{a}$

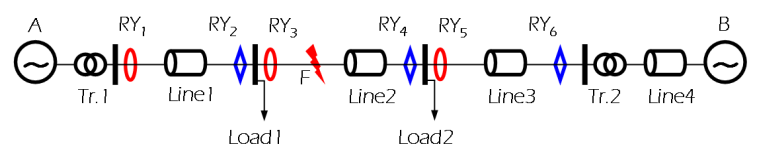

Fig. 5 Single line diagram of target system
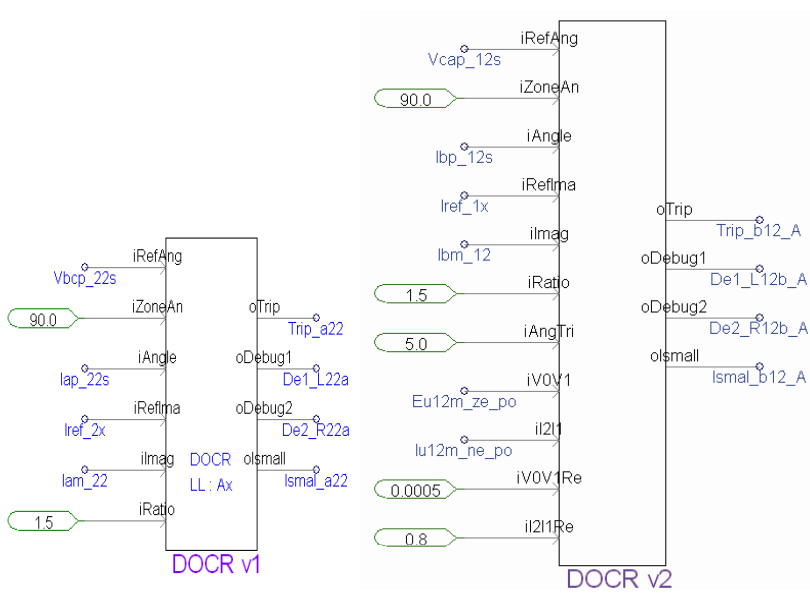

Fig. 6 DOCR model v1 and v2
Table 2. Pin description of DOCR modules(v1, v2)

\begin{tabular}{c|l|c|c}
\hline In/Output & \multicolumn{1}{|c|}{ Description } & \multicolumn{2}{|c}{ Adoption } \\
\cline { 3 - 4 } name & & v1 & v2 \\
\hline iRefAng & Reference Angle(polarization quantity) & $\sqrt{ }$ & $\sqrt{ }$ \\
\hline iZoneAng & Assign the trip region to DOCR & $\sqrt{ }$ & $\sqrt{ }$ \\
\hline iAngle & phase of L-L fault current & $\sqrt{ }$ & $\sqrt{ }$ \\
\hline iRefImag & Reference for Magnitude of fault current & $\sqrt{ }$ & $\sqrt{ }$ \\
\hline ilmag & Magnitude of L-L fault current & $\sqrt{ }$ & $\sqrt{ }$ \\
\hline iRatio & Ratio to detect the fault by magnitude & $\sqrt{ }$ & $\sqrt{ }$ \\
\hline iAngTrim & trip region controlling value & - & $\sqrt{ }$ \\
\hline V0V1 & magnitude of $\mathrm{V}_{0} / \mathrm{V}_{1}$ & - & $\sqrt{ }$ \\
\hline iI2I1 & magnitude of $\mathrm{I}_{2} / \mathrm{I}_{1}$ & - & $\sqrt{ }$ \\
\hline iV0V1Ref & Reference for magnitude of $\mathrm{V}_{0} / \mathrm{V}_{1}$ & - & $\sqrt{ }$ \\
\hline iI2I1Ref & Reference for magnitude of $\mathrm{I}_{2} / \mathrm{I}_{1}$ & - & $\sqrt{ }$ \\
\hline oTrip & Trip value & $\sqrt{ }$ & $\sqrt{ }$ \\
\hline oDebug & For debugging & $\sqrt{ }$ & $\sqrt{ }$ \\
\hline oIsmall & Flag to show the magnitude of fault current & $\sqrt{ }$ & $\sqrt{ }$ \\
\hline
\end{tabular}

\subsection{Simulation result of conventional DOCR model} (v1)

Operation characteristic of DOCR in L-L fault of point F of Fig. 5 is analyzed. The scope of the resistance value of fault impedance $(Z=R+j 0.0377)$ is $1 \sim 10[\Omega]$. Table 3 shows operation result of RY1 6, and Table 4 shows the magnitude-phase angles and reference phase angles of fault current $\mathrm{I}_{\mathrm{ab}}$ of RY1. The initial trip region $\left(\theta_{2}-\theta_{1}\right)$ is $180\left[^{\circ}\right]$ in the simulation.

In the given system condition, up to $7[\Omega]$, DOCR protection cooperation of the system is perfect, in the Fig. 7 , the phase of fault current is in the trip region. But abnormality is occurred from $8[\Omega]$. As shown in the Table 3 , operational characteristics of installed DOCR is not consistent, and in case of RY1, mal-operation is occurred

Table 3. Summary of DOCR operation

\begin{tabular}{c|c|c|c|c|c|c|c|c|c|c}
\hline & $1[\Omega]$ & $2 \Omega]$ & $3 \Omega]$ & $4 \Omega]$ & $5 \Omega]$ & $6 \Omega]$ & $7 \Omega]$ & $8 \Omega]$ & $9 \Omega]$ & $10 \Omega]$ \\
\hline RY1 & $\circ$ & $\circ$ & $\circ$ & $\circ$ & $\circ$ & $\circ$ & $\circ$ & $\times$ & $\times$ & $\times$ \\
\hline RY3 & $\circ$ & $\circ$ & $\circ$ & $\circ$ & $\circ$ & $\circ$ & $\times$ & $\times$ & $\times$ & $\times$ \\
\hline RY4 & $\square$ & $\square$ & $\square$ & $\square$ & $\square$ & $\square$ & $\square$ & $\square$ & $\square$ & $\square$ \\
\hline RY2 & $\square$ & $\square$ & $\square$ & $\square$ & $\square$ & $\square$ & $\square$ & $\triangle$ & $\triangle$ & $\triangle$ \\
\hline RY4 & $\circ$ & $\circ$ & $\times$ & $\times$ & $\times$ & $\times$ & $\times$ & $\times$ & $\times$ & $\times$ \\
\hline RY6 & $\circ$ & $\circ$ & $\times$ & $\times$ & $\times$ & $\times$ & $\times$ & $\times$ & $\times$ & $\times$ \\
\hline
\end{tabular}

$\circ$ : correct trip(operation that trips in status that shall be trip)

$\square$ : correct restrain(operation that does not trip in restrainable status)

$\times$ : mal-operation(operation that restrains in status that shall be trip)

$\triangle$ : mis-operation(operation that trips in status that shall be restrained)

Table 4. Summary of numerical results of $\mathrm{I}_{\mathrm{ab}}$ of RY1

\begin{tabular}{c|c|c|c|c|c|c|c|c|c|c}
\hline & $1[\Omega]$ & $2 \Omega]$ & $3 \Omega]$ & $4 \Omega]$ & $5 \Omega]$ & $6 \Omega]$ & $7 \Omega]$ & $8 \Omega]$ & $9 \Omega]$ & $10 \Omega]$ \\
\hline $\mathrm{Op}$ & $\circ$ & $\circ$ & $\circ$ & $\circ$ & $\circ$ & $\circ$ & $\circ$ & $\times$ & $\times$ & $\times$ \\
\hline $\mathrm{kA}$ & 5.5 & 4.9 & 4.3 & 3.7 & 3.3 & 2.9 & 2.6 & 2.4 & 2.2 & 2.0 \\
\hline$\theta\left[^{\circ}\right]$ & 9.1 & 13.4 & 15.9 & 17.4 & 18.3 & 19.0 & 19.4 & 19.7 & 19.8 & 19.9 \\
\hline$\theta_{1}\left[^{\circ}\right]$ & 72.2 & 57.7 & 44.9 & 35.3 & 27.4 & 23.5 & 22.9 & 22.2 & 20.1 & 18.5 \\
\hline$\theta_{2}\left[^{\circ}\right]$ & 252.2 & 237.7 & 224.9 & 215.3 & 208.4 & 203.5 & 199.9 & 192.2 & 195.1 & 193.5 \\
\hline & : phase of fault current $\left(\mathrm{I}_{\mathrm{ab}}\right)$ \\
$\theta_{1}:$ Starting angle of non-trip region in Fig. 7, 8 & \\
$\theta_{2}:$ Ending angle of non-trip region in Fig. 7, 8
\end{tabular}




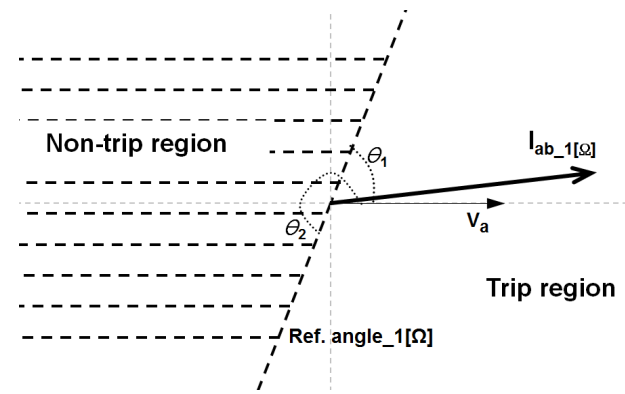

Fig. 7 Locus of $I_{a b}$ of DOCR RY1, correct trip

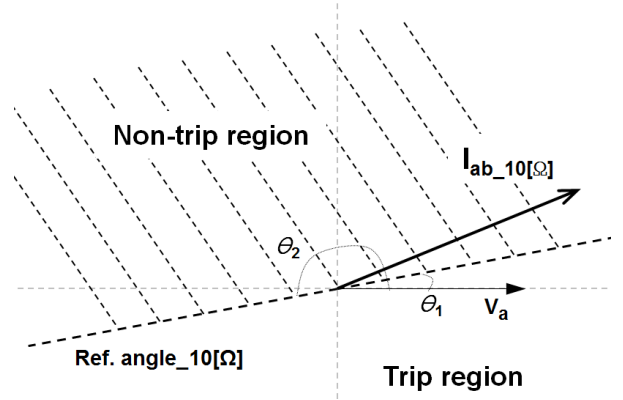

Fig. 8 Locus of $\mathrm{I}_{\mathrm{ab}}$ of DOCR RY1, mal-operation

from the high resistance value, and in case of RY4.RY6, mal-operation is occurred from the low resistance value. The main reason that operational characteristics are not consistent is that positive-sequence, negative-sequence impedance from the view of fault location are different. Also, the range between the phase angles of fault current shown from the relay operated on the weak power system, and trip region is relatively small. As a result, the more fault resistance value increases, the more phase of fault current deviates the protection zone. As shown in the Fig. 8, the phase of fault current of high impedance condition $(10[\Omega])$ fell into the non-trip region.

In Eq. (1) and (2), when L-L fault occurs, magnitude phase of fault current are determined by only with sequence impedance and fault impedance, so followings are able to be checked.

- Contact with trees, trespass of small animal, input of single phase heavy load are basically not able to be classified with L-L fault.

- For more accurate operation of DOCR relay, reflection of sequence impedance of installed position is required.

\section{Mal-operation Reduction Algorithm}

\subsection{Mal-operation reduction algorithm}

The Algorithm reducing mal-operation of DOCR is to add ratio of positive-sequence voltage current, and negativesequence voltage-current to the identification factor of

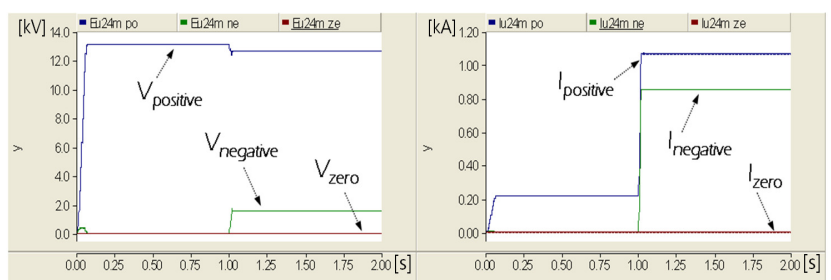

Fig. 9. Profile of sequence component of V'I at RY1.

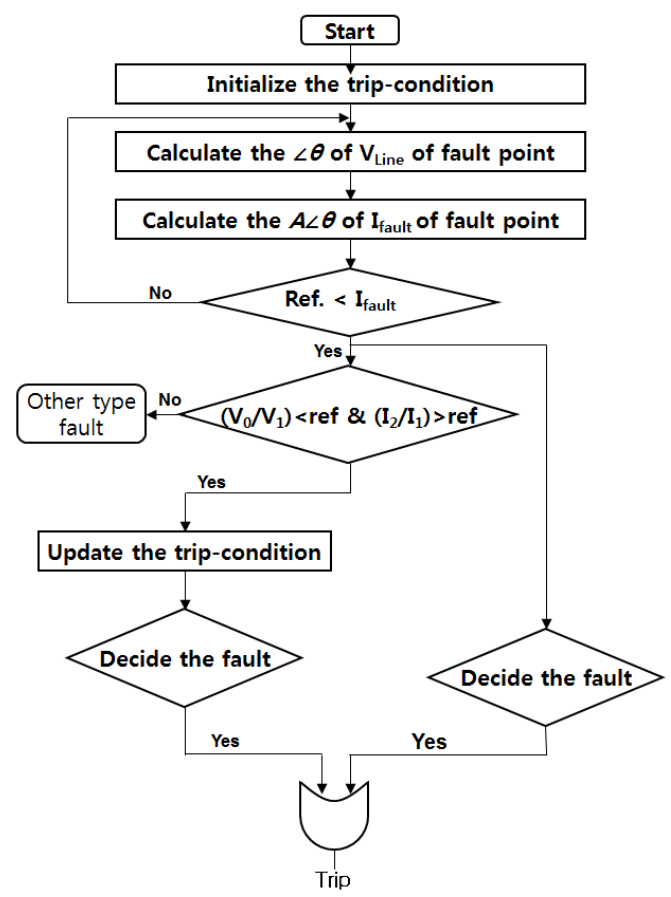

Fig. 10 Flowchart of advanced DOCR Algorithm

DOCR, and if the ratio value reaches specific magnitude, it shall extend the trip region. In field application, initial trip region is $170 \sim 180\left[^{\circ}\right]$. The Fig. 9 shows the magnitude of each component of voltage-current value calculated from RY1(fault at 1[s]). Positive-sequence and negative-sequence are changed before and after the fault, but zero-sequence always shows 0 , so it is confirmed that to use it as an identification factor of DOCR is proper.

Fig. 10 is an operation algorithm flow chart of the proposed DOCR. As shown in the Fig. 9, $\mathrm{V}_{0} / \mathrm{V}_{1}, \mathrm{I}_{2} / \mathrm{I}_{1}$ are selected as a ratio of each component of voltage current. In case of L-L fault, when $\mathrm{V}_{0} / \mathrm{V}_{1}$ is less than reference value, and $I_{2} / I_{1}$ is more than reference value, these are identified as L-L fault(If the fault type is SLG, zero sequence V, I are the dominant component). And a certain part of non-trip region is reduced to avoid mal-operation as much as possible. Also, a conventional method that identifies by the magnitude and phase angles of fault current and the proposed algorithm is applied in parallel in order to assure operation stability of protective relay. As the digital relay that is recently and mainly applied to the system calculates voltage-current values via numerical calculation from internal DSP(Digital Signal Processor) or CPU (Central 


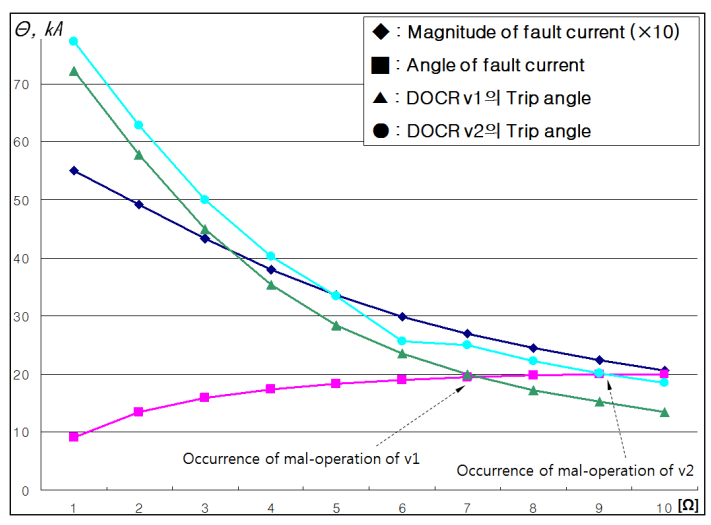

Fig. 11. $I_{a b}$, v1 Trip angle, v2 Trip angle of RY1

Table 5. Summary of numerical results of $I_{a b}$ of RY1

\begin{tabular}{c|c|c|c|c|c|c|c|c|c|c}
\hline & $1[\Omega]$ & $2 \Omega]$ & $3 \Omega]$ & $4 \Omega]$ & $5 \Omega]$ & $6 \Omega]$ & $7 \Omega]$ & $8 \Omega]$ & $9 \Omega]$ & $10 \Omega]$ \\
\hline $\mathrm{Op}$ & $\circ$ & $\circ$ & $\circ$ & $\circ$ & $\circ$ & $\circ$ & $\circ$ & $\bigcirc$ & $\bigcirc$ & $\times$ \\
\hline $\mathrm{kA}$ & 5.5 & 4.9 & 4.3 & 3.7 & 3.3 & 2.9 & 2.6 & 2.4 & 2.2 & 2.0 \\
\hline$\left.\theta^{\circ}\right]$ & 9.1 & 13.4 & 15.9 & 17.4 & 18.3 & 19.0 & 19.4 & 19.7 & 19.8 & 19.9 \\
\hline$\theta_{1}\left[^{\circ}\right]$ & 72.2 & 57.7 & 44.9 & 40.3 & 33.4 & 25.5 & 24.9 & 22.2 & 20.1 & 18.5 \\
\hline$\theta_{2}\left[^{\circ}\right]$ & 252.2 & 237.2 & 219.9 & 210.3 & 203.4 & 198.5 & 194.9 & 192.2 & 190.1 & 188.5 \\
\hline
\end{tabular}

Processing Unit), new proposed DOCR relay model can be easily implemented. Users can naturally disable the new proposed algorithm.

\subsection{Simulation Result of advanced DOCR model(v2)}

The result of DOCR relay of RY1 using proposed algorithm is as Fig. 11, and Table 5. As a result of using proposed algorithm, occurrence of mal-operation is reduced, and reliability of protective relay is confirmed to increase. Fig. 11 shows phase angles of fault current and reference phase angles in conventional DOCR model. As described above, the more fault resistance value increases, the more it is approached to non-trip region, and consequently maloperation is able to be confirmed Fig. 8.

\section{Conclusion}

In this paper, the DOCR operation characteristic in bidirectional power flow system is explained via theory and PSCAD simulation. For the simulation, because PSCAD don't support the DOCR model, two types of DOCR relay model are newly developed. Through this paper, the causes of mal-operation of DOCR is analyzed and to complement, the new DOCR operation algorithm using the positive. negative sequence value and controlling the trip region is proposed. As a result of simulation, it is confirmed that the proposed algorithm decreases possibility of mal-operation of protective relay, and contributes to stable operation of the system including DG. Generally, mal-operation is more severe situation than mis-operation, because mal-operation of relay causes a great deal of damage to the utilities itself and economical loss to the operator. Also, using proposed relay model, the protection coordination of bi-directional or DG system can be analyzed in more detail.

To reduce the mal-operation, this paper decreases the non-trip region by $5.5 \%$. Future work would investigate the optimal decreasing value and the protection coordination of DG.

\section{Acknowledgements}

This work was supported by "Human Resources Program in Energy Technology" of the Korea Institute of Energy Technology Evaluation and Planning(KETEP), granted financial resources from the Ministry of Trade, Industry \& Energy, Republic of Korea. (No. 20144030200450)

\section{References}

[1] J. M. Gers and E. J. Holmes, Protection of Electricity Distribution Networks - 2nd Edition, London: The Institution of electrical Engineers, 2005

[2] Jae-Eon. Kim, Modelling \& Analysis for Distribution systems with Distributed Generation, Seoul : Naeha Press, 2012

[3] KEPCO, Business Guideline of Management Procedure for $D G, 2012$

[4] S. Voima, K. Kauhaniemi, H. Laaksonen, "Novel protection approach for mv microgrid," CIRED 21st International Conference on Electricity Distribution Frankfurt, 6-9 June 2011

[5] N. Nimpitiwan and G. Heydt, "Consequences of Fault Currents Contributed by Distributed Generation," PSERC Publication 06-21, June 2006

[6] G. Buigues, et al., "Microgrid Protection: Technical challenges and existing techniques," International Conference on Renewable Energies and Power Quality (ICREPQ’13) Bilbao (Spain), March, 2013

[7] IEEE PES Power System Relaying Committee, "Considerations in Choosing Directional Polarizing Methods for Ground Overcurrent Elements in Line Protection Applications," MAY 2014

[8] Protection System Misoperations Task Force, "Page 26, Misoperations Report" NERC Planning Committee on April 8, 2013

[9] W. K. Sonnemann, A Study of Directional Element Connections for Phase Relays, AIEE, 1950.

[10] M. Yadav, C. Gupta, et al., "Optimal Coordination of Directional Overcurrent Relay: A Review," International Journal of Emerging Technology and Advanced Engineering, Volume 4, Issue 5, May 2014

[11] A. Ukil, B. Deck, et al., "Current-Only Directional Overcurrent Relay," IEEE SENSORS JOURNAL, 
2010

[12] L. Susilo, J. C. Gu and S. K. Huang, "Fault current Characterization Based on Fuzzy Algorithm for DOCR Application," Energy and Power Engineering, 2013, 5, 932-936

[13] A. Wixon, et al, "Load blinding stabilizations for DOC relays subjected to reverse load flow," Alstom

[14] D. Yuan, et al, "An New Directional Comparison Scheme for Distribution Line Protection," Universities Power Engineering Conference, Volume 3, September 2006

[15] William D. Stevenson, Jr, Elements of Power system Analysisis, $4^{\text {th }}$ edition, Singapore : McGRAW-HILL Press

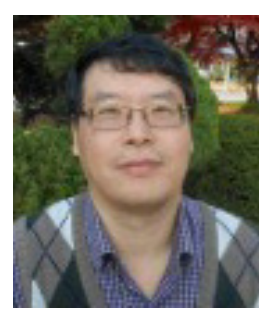

Su-Hyeong Jang was born in Seoul, KOREA, in 1968. He received his B.S. and M.S. degrees in Electrical Engineering from Hanyang Univ., in 1991 and 1994, respectively. He is presently pursuing toward his Ph.D. degree at Chungbuk National Univ. Since 1994, He has been with the R\&D Lab., LS Industrial Systems, is currently principle research engineer. His research interests include protective relay, WAMS based by PMU, Distributed Generation and Electro Magnetic Compatibility of IED.

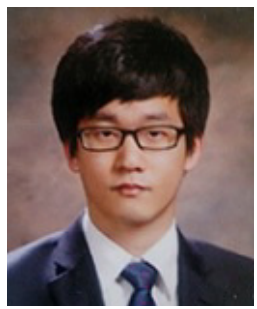

Joon-Seok Oh He received the B.S. degree in Electric Engineering from Chungbuk National University, Korea, in 2014. He is currently working toward his M.S. in Electric Engineering at Chungbuk National University. His research interests include operation and design of power distribution systems with distributed generation.

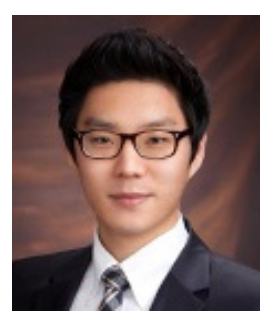

Ui-Yong Jeong He received the B.S. degree in Electric Engineering from Chungbuk National University, Korea, in 2014. He is currently working toward his M.S. in Electric Engineering at Chungbuk National University. His research interests include operation and design of power distribution systems with distributed generation.

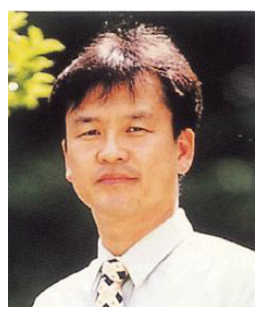

Jae-Eon Kim $\mathrm{He}$ received the B.S. and M.S. degrees from the University of Hanyang in 1982 and 1984, respectively. He was affiliated with KERI as a researcher from 1984 to 1989; a senior researcher form 1989 to 1996; and a team leader of advanced distribution systems and custom power lab from 1997 to 1998. He received his Ph.D. from Kyoto University, Japan in 1996. He has been a professor at Chungbuk National University since 1998. His current interests are analysis of power quality; operation and design of power distribution systems with distributed generation and advanced distribution systems, such as micro-grid or smart grid.

\section{Appendix 1.}

$$
\begin{aligned}
& I_{a b}=\frac{\left(1-a^{2}\right) V_{a}}{Z_{1}+Z_{2}+Z_{f}}=\frac{\left(1-a^{2}\right) V_{a}}{\left(R_{1}+R_{2}+R_{f}\right)+j\left(X_{1}+X_{2}+X_{f}\right)} \\
& \text { Let } M=R_{1}+R_{2}+R_{f}, N=X_{1}+X_{2}+X_{f} \\
& I_{a b}=\frac{\left(1-a^{2}\right) V_{a}}{M+j N}=\frac{V_{a}\left(1-a^{2}\right)(M-j N)}{(M+j N)(M-j N)} \\
& =\frac{V_{a}\left(1-a^{2}\right)(M-j N)}{M^{2}+N^{2}} \\
& =\frac{V_{a}\left(1-0.5-j \frac{\sqrt{3}}{2}\right)(M-j N)}{M^{2}+N^{2}} \\
& =\frac{V_{a}\left(0.5 M-\frac{\sqrt{3}}{2} N-j\left(0.5 N+\frac{\sqrt{3}}{2} M\right)\right) \times 2}{\left(M^{2}+N^{2}\right) \times 2} \\
& =\frac{V_{a}(M-\sqrt{3} N-j(N+\sqrt{3} M))}{\left(M^{2}+N^{2}\right) \times 2} \\
& =A \angle \theta \\
& \therefore A=\frac{V_{a} \sqrt{G^{2}+H^{2}}}{2\left(M^{2}+N^{2}\right)} \quad \theta=\tan ^{-1}\left(\frac{-(N+\sqrt{3} M)}{M-\sqrt{3} N}\right) \\
& \text { here, } \quad G=(M-\sqrt{3} N) \quad H=(N+\sqrt{3} M)
\end{aligned}
$$




\section{Appendix 2.}

A2.1) Tr. 1 specification

\begin{tabular}{c|c}
\hline Item & Specification \\
\hline Voltage, primary & $154[\mathrm{kV}]$ \\
\hline Voltage, secondary & $22.9[\mathrm{kV}]$ \\
\hline Voltage, tertiary & $6.6[\mathrm{kV}]$ \\
\hline
\end{tabular}

A2.2) Tr. 2 specification

\begin{tabular}{c|c}
\hline Item & Specification \\
\hline Voltage, primary & $154[\mathrm{kV}]$ \\
\hline Voltage, secondary & $22.9[\mathrm{kV}]$ \\
\hline Leakage reactance & $0.09[\mathrm{pu}]$ \\
\hline
\end{tabular}

A2.3) Load specification

\begin{tabular}{c|c}
\hline Item & Specification \\
\hline Load 1 & $9.0[\mathrm{MW}]$ \\
\hline Load 2 & $1.0[\mathrm{MW}]$ \\
\hline
\end{tabular}

A2.4) Line specification

\begin{tabular}{c|c|c|c|c}
\hline & Type & Length $[\mathrm{m}]$ & $\mathrm{R}[\mathrm{pu} / \mathrm{km}]$ & $\mathrm{X}[\mathrm{pu} / \mathrm{km}]$ \\
\hline Line 1 & CNCV325 & 1000 & 0.0000165 & 0.0000181 \\
\hline Line 2 & ACSR160 & 700 & 0.0008705 & 0.0023858 \\
\hline Line 3 & $"$ & 300 & $"$ & $"$ \\
\hline Line 4 & $"$ & 3000 & $"$ & $"$ \\
\hline
\end{tabular}

Appendix 3.

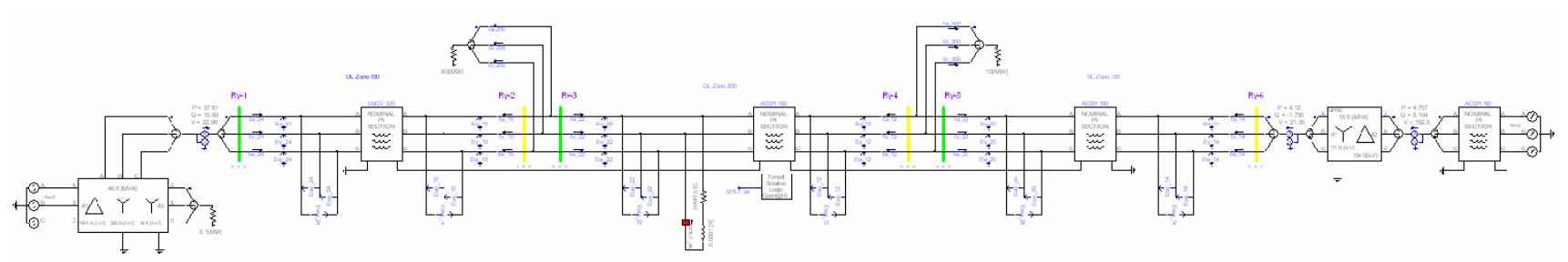

